\title{
Akupunktur gegen menopausale Hitzewallungen?
}

\author{
Einige - wenige - Studien haben \\ einen positiven Effekt der konventio- \\ nellen Akupunktur auf menopausale \\ Beschwerden gezeigt. Ob dieser \\ Effekt auch mit der nicht invasiven \\ Laserakupunktur zu erreichen ist?
}

- Forscher aus Neuseeland haben 40 Frauen mit menopausalen Hitzewallungen entweder mit Laserakupunktur oder mit Scheinlaser zwölf Wochen lang behandelt. Bei Behandlungsende waren die Hitzewallungen und andere Symptome deutlich reduziert - sowohl in der Experimental- als auch in der Kontrollgruppe.

Ein signifikanter Gruppenunterschied bestand nicht. Wenn überhaupt, so waren die Ergebnisse unter Scheinlaser marginal besser. Die Autoren schließen daraus, das die Laserakupunktur bei dieser Indikation nicht effektiv ist.

\section{Kommentar}

Die Studie ist aus verschiedenen Gründen interessant. Die Punktwahl wurde nach TCM-Prinzipien getroffen. Die Therapie

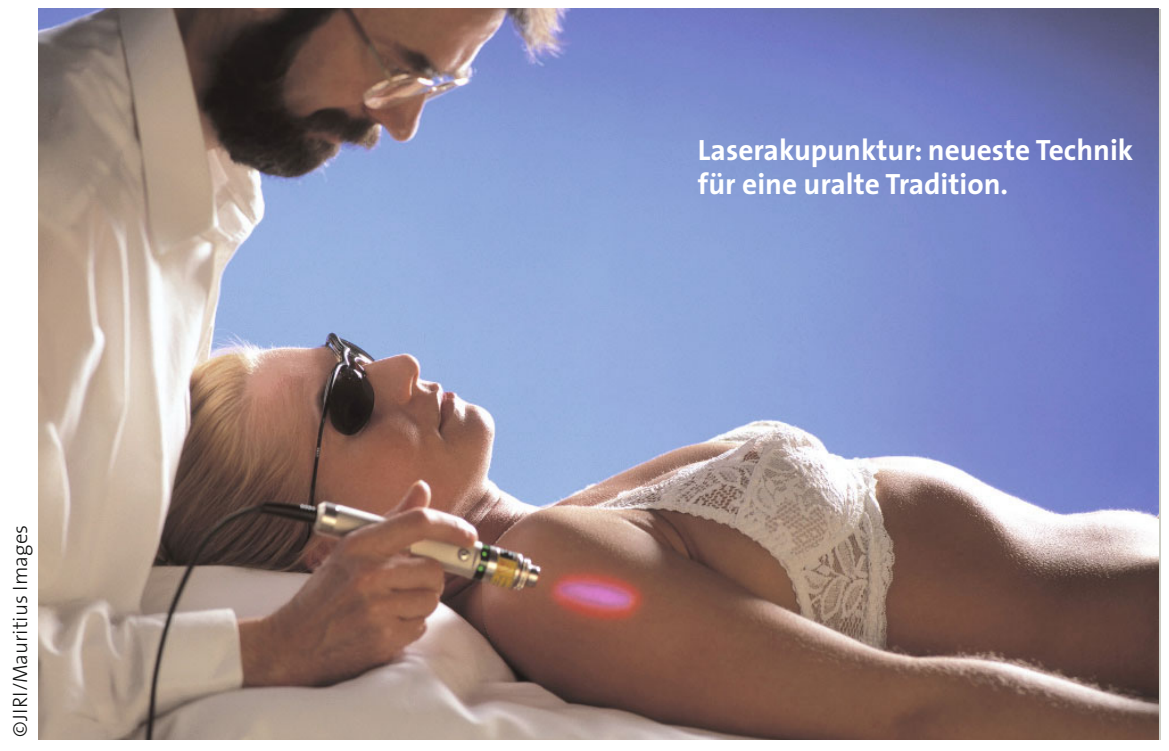

verbindet also uralte Tradition mit neues-ter Technik (Laser). Daher ist ein nicht unerheblicher Placeboeffekt zu erwarten. Tatsächlich zeigt dieser sich auch in dem guten Ansprechen beider Gruppen. Ich kenne viele Proponenten der Komplementärmedizin, die daraus schließen würden, dass deshalb diese Art der Therapie zu befürworten sei. Ich dagegen meine, dass uns diese Denkweise im Laufschritt zurück ins medizinische Mittelalter führen würde.

E. ERNST :

- K. A. O'Brien et al.

Laser acupuncture does not improve menopausal symptoms. Menopause 2010; doi: 10.1097/gme.obo13e3181c72bgd

\section{Wie Fettsucht das Nierensteinrisiko erhöht}

\begin{abstract}
Mehrere epidemiologische Studien haben eine starke Beziehung zwischen Fettsucht und Nierensteinen gezeigt. Ob das Risiko, an Nierensteinen zu erkranken, vom Ausmaß der Adipositas abhängig ist, war Gegenstand einer aktuellen Studie.
\end{abstract}

— Forscher der Johns Hopkins University werteten die Daten von 95598 Personen einer privaten Versicherung aus. Die Inzidenz von Nierensteinen in Personen mit oder ohne Fettsucht sowie die Odds Ratios (OR) wurden berechnet. Als Fettsucht wurde ein BMI > $30 \mathrm{~kg} / \mathrm{m}^{2}$ Körperoberfläche definiert.

3257 Steinbildner wurden gefunden: 42,9\% Männer und 57,1\% Frauen. Unter den nicht fettsüchtigen Per- sonen wurden 2,6\% Steinträger diagnostiziert. Fast doppelt so hoch $(4,9 \%)$ war der Anteil der Steinträger unter den adipösen Personen.

Eine signifikante Assoziation zwischen BMI und Nierensteinen bestand nur bei einem BMI > $30 \mathrm{~kg} / \mathrm{m}$. Wurden die BMIs der fettsüchtigen Gruppe von 30 bis $>50 \mathrm{~kg} / \mathrm{m}^{2}$ sortiert, dann blieb das erhöhte Steinrisiko konstant, unabhängig davon wie schwer die Personen waren.

\section{Kommentar}

Ein $B M I>30$ ist mit einem erhöhten Nierensteinrisiko assoziiert. Dieses Risiko scheint dann unabhängig von einer weiteren Gewichtzunahme stabil zu sein, denn noch höhere BMI-Werte gingen nicht mit einem weiter erhöhten Steinrisiko einher. Sicher kommen noch Diät-, metabolische und endokrine Faktoren hinzu, die in zukünftigen Studien untersucht werden sollten.

K. MALBERG = 